Fig. 1 Reverse IV characteristics in dark conditions for $1 \mathrm{x} 1 \mathrm{~mm}^{2}$ detector.

Fig. 2 Typical MPPC pulse with its main characteristics.

Fig. 3 Gain vs. $\mathrm{V}_{\text {bias }}$ at different $\mathrm{T}$ for $1 \times 1 \mathrm{~mm}^{2}$ detector.

Fig. $4 \mathrm{~V}_{\mathrm{BD}}$ vs. $\mathrm{T}$ for $1 \mathrm{x} 1$ and $3 \times 3 \mathrm{~mm}^{2}$ detectors.

Fig. 5 Gain vs. $\Delta V$ for a) $1 \times 1 \mathrm{~mm}^{2}$ and b) $3 \times 3 \mathrm{~mm}^{2}$ detectors.

Fig. $6 \mathrm{C}_{\mu \text { cell }}$ vs $\mathrm{T}$ for $1 \mathrm{x} 1$ and $3 \times 3 \mathrm{~mm}^{2}$ detectors.

Fig. 7 Normalized MPPC signal shapes vs. T for a) $1 \times 1 \mathrm{~mm}^{2}$ and b) $3 \times 3 \mathrm{~mm}^{2}$ detectors.

Fig. $8 \mathrm{R}_{\mathrm{q}}$ vs. T for $1 \times 1 \mathrm{~mm}^{2}$ and $3 \times 3 \mathrm{~mm}^{2}$ detectors.

Fig. 9 DCR vs. $\Delta V$ for a) $1 \times 1 \mathrm{~mm}^{2}$ and b) $3 \times 3 \mathrm{~mm}^{2}$ detectors. 


\title{
STUDIES OF MPPC DETECTORS DOWN TO CRYOGENIC
} TEMPERATURES

\author{
N. Dinu ${ }^{a}$, A. Nagai ${ }^{a^{1}}$, A.Para ${ }^{b}$ \\ a Laboratory of Linear Accelerator \& University Paris XI, CNRS / IN2P3, 91898 Orsay Cedex, France \\ b Fermi National Accelerator Laboratory, Batavia Il, 60510-5011 USA
}

Keywords: Silicon PhotoMultiplier, breakdown voltage, gain, dark count rate, capacitance, signal shape

Abstract

This work reports on the behavior of the Multi-Pixel Photon Counter (MPPC) detectors produced by Hamamatsu HPK in a temperature range from $+55^{\circ} \mathrm{C}$ down to $-175^{\circ} \mathrm{C}$. Devices of $1 \times 1$ $\mathrm{mm}^{2}$ and $3 \times 3 \mathrm{~mm}^{2}$ total area and $50 \times 50 \mu \mathrm{m}^{2} \mu$ cell size have been studied. Electrical parameters as breakdown voltage, gain, capacitance, pulse shape, quenching resistance and dark count rate were measured and some of them showed important temperature variation. Besides of this temperature dependence, it is shown that MPPC detectors can operate with a stable gain independent of $\mathrm{T}$ if the overvoltage is kept constant. Moreover, at a given temperature, the device of $3 \times 3 \mathrm{~mm}^{2}$ (production year 2011) presents seven times less dark rate $/ \mathrm{mm}^{2}$ with respect to the one of $1 \mathrm{x} 1 \mathrm{~mm}^{2}$ (production year 2007), showing an important technological improvement implemented by HPK during four year's time interval.

\section{Introduction}

Nowadays, the MPPC detector (also referred to us Silicon PhotoMultiplier) has become an alternative photon detector for many applications that would employ typically PMTs or MA-PMTs [1, 2]. Their important advantages as compactness, fastness, insensibility to magnetic fields, high gain, and low operating voltage make them very attractive in medical imaging applications for diagnostic and therapeutic purposes as well as for high energy physics or astrophysics experiments. However, in any of these applications where usually multi-photon detection is involved, some important electrical parameters as breakdown voltage or pulse shape present significant temperature variations. Therefore, a study of these parameters in a wide temperature range from $+55^{\circ} \mathrm{C}$ down to cryogenic temperatures allows us to have a closer look to the physical phenomena staying behind this temperature dependence and eventually find equivalent operating conditions independent of temperature. Moreover, state of the art sensitivity in light detection can be reached by operating MPPCs at low temperatures. There, single photon detection can be achieved and consequently a whole range of potential applications is opened, from particle detection with noble liquids to Cerenkov light detection. In this paper, we report our studies on the electrical behavior of two MPPC detectors produced by Hamamatsu HPK in a temperature range from $+55^{\circ} \mathrm{C}$ down to $-175^{\circ} \mathrm{C}$.

\footnotetext{
${ }^{1}$ Part of the presented work was done during the Physics Master performed at the Nuclear Physic Department of Taras Shevchenko National University of Kiev, 01601 Kiev, Ukraine.
} 
Two experimental set-ups have been used for the measurements presented in this paper: one based on a climatic chamber working within a temperature $\mathrm{T}$ range of $+35>\mathrm{T}>-5^{\circ} \mathrm{C}$, and a second one implying a cryogenic system working in a $\mathrm{T}$ range of $+55>\mathrm{T}>-175^{\circ} \mathrm{C}$.

The first set-up allowed the static characterization of MPPC detectors (i.e. current-voltage characteristic) in the dark. During measurements, the detectors have been connected to a Keithley 2611 source-meter for bias voltage supply and current measurement. The $\mathrm{T}$ was controlled by the climatic chamber and complementary monitored by a Pt100 sensor readout by a Keithley 2000 multimeter.

The second set-up was used for the dynamic characterization of MPPC detectors. The device under test has been illuminated with very short light pulses given by a pulsed laser through an optical fiber. The voltage of the laser has been adjusted in such way that only a few photons per pulse were generated.

During dynamic measurements, the detectors have been located in a small box of $\sim 30 \times 30 \times 30 \mathrm{~cm}^{2}$ called test-cube, where the $\mathrm{T}$ has been assured by a system of fully automatic $\mathrm{N}_{2}$ fulfilling and temperature control. A copper rod, having one extremity in contact with tested MPPC and other one in contact with a vessel filled with $\mathrm{N}_{2}$, serves as a cold finger used to cool down the samples. A Pt100 temperature sensor and a resistive heater were mounted close to detector to control and stabilize the temperature. To reduce a heat exchange, the test cube has been carried out under vacuum conditions at $\mathrm{P} \sim 5 \times 10^{-3} \mathrm{mb}$.

The read-out and data acquisition system during dynamic tests consisted of a Miteq amplifier (gain $=50 \mathrm{~dB}$, bandwidth $=500 \mathrm{MHz}$ ), followed by an Agilent oscilloscope for waveforms acquisition (a bandwidth of $20 \mathrm{MHz}$ has been used to reduce the influence of the electronic noise). A Keithley 2400 was used for MPPC bias supply. All used electronics have been located outside the test cube, at a room temperature, to provide the same experimental conditions (amplifier gain, electronic noise level) independent of $\mathrm{T}$.

Two MPPC devices produced by Hamamatsu HPK have been tested:

- MPPC S10362-11-050U-3, with a total area of $1 \mathrm{x} 1 \mathrm{~mm}^{2}$ and a $\mu$ cell size of $50 \times 50 \mu \mathrm{m}^{2}$ (i.e. $400 \mu$ cells) in metal package, produced in 2007 ;

- MPPC S10931-33-050P-63813, with a total area of $3 \times 3 \mathrm{~mm}^{2}$ and $\mu$ cell size of 50x50 $\mathrm{mm}^{2}$ (i.e. $3600 \mu$ cells) in plastic package, produced in 2011 year.

Experimental data have been acquired at different temperatures $\mathrm{T}$ ranging from $+55^{\circ} \mathrm{C}$ down to $175^{\circ} \mathrm{C}$ in steps of $10^{\circ} \mathrm{C}$. At each temperature, the device has been operated at twelve biases voltages $\mathrm{V}_{\text {bias. }}$. Previous measurements performed on MPPC S10362-11-050U-3 detector [3] showed that its breakdown voltage $\mathrm{V}_{\mathrm{BD}}$ is $\sim 68.65 \mathrm{~V}$ at $25^{\circ} \mathrm{C}$ and it decreases with $\mathrm{T}$ of $\sim 58 \mathrm{mV} /{ }^{\circ} \mathrm{C}$. Similar values of $\mathrm{V}_{\mathrm{BD}}$ were supposed for the second detector. Therefore, to measure the devices in comparable operating conditions independent of $T$, each $V_{\text {bias }}$ has been selected to obtain the same overvoltage $\left(\Delta V=V_{\text {bias }}-\right.$ $\mathrm{V}_{\mathrm{BD}}$ ) with respect to $\mathrm{V}_{\mathrm{BD}}$. In such way, at each $\mathrm{T}$, every detector has been operated in a $\Delta \mathrm{V}$ range from $0.5 \mathrm{~V}$ to $3 \mathrm{~V}$. For every $\mathrm{V}_{\text {bias }}, 5000$ waveforms have been saved, each waveform of $5 \mu \mathrm{s}$ length. The acquisition of the waveforms has been triggered by an electrical signal given by the laser.

\section{Static characterization}


The reverse IV characteristics of $1 \mathrm{x} 1 \mathrm{~mm}^{2}$ detector at different $\mathrm{T}$ are presented in Fig.1. It is well know today $[1,2]$ that the MPPC device is represented by a parallel array of many $\mu$ cells, where each $\mu$ cell is a pn junction working in reverse mode connected in series with a quenching resistance. Therefore, it is evident that the knee above which the reverse leakage current increases very rapidly with $\mathrm{V}_{\text {bias }}$ is related to the device breakdown. The $\mathrm{V}_{\mathrm{BD}}$ decreases with $\mathrm{T}$ as expected, due to increase of ionization rates at low $\mathrm{T}[4,5]$. However, the shape of IV curves is device dependent (i.e. geometry, technology) and a standard method allowing an absolute determination of $\mathrm{V}_{\mathrm{BD}}$ value is quite difficult to identify. The method of second derivative of the logarithm of the current has been reported for similar detectors [6]. Using this mathematical method, the $\mathrm{V}_{\mathrm{BD}}$ of $1 \times 1 \mathrm{~mm}^{2}$ detector has been calculated. The results are reported later in this article (see section 4.2), when a comparison with $V_{B D}$ values determined from the analysis of dynamic parameters will be also given.

Fig. 1 Reverse IV characteristics in dark conditions for $1 \times 1 \mathrm{~mm}^{2}$ detector.

\section{Dynamic characterization}

To analyze the huge amount of experimental data acquired in dynamic mode ( 24 values of $\mathrm{T}, 12$ values of $\mathrm{V}_{\text {bias }}$ for each $\mathrm{T}$ and 5000 waveforms per $\mathrm{V}_{\text {bias }}$, leading to $1.44 \cdot 10^{6}$ waveforms per detector), an automatic procedure for data analysis, based on ROOT data Analysis Framework [7], has been developed.

This procedure uses experimental waveforms from oscilloscope as input files and creates output Ntuples files with MPPC pulses characteristics. Its main steps are:

- baseline restoration to eliminate the differentiation of the waveform determined by the readout-electronics and to restore its zero baseline;

- template creation to calculate the typical MPPC pulse shape at a given T;

- pulse finding procedure to determine the time intervals containing MPPC pulses (i.e. "single pulse" " or "train of pulses" $)$;

- template subtraction to reconstruct all MPPC pulses in a "train of pulses";

- analysis of MPPC pulse characteristics.

Based on this procedure, MPPC pulse characteristics such as baseline, time position and amplitude, rise time and recovery time, charge $Q$ and chi-square $\chi^{2}, t_{\text {before }}{ }^{2}$ and $t_{\text {after }}{ }^{3}$, for each experimental condition ( $\mathrm{T}$ and $\mathrm{V}_{\text {bias }}$ ) were determined. All these characteristics are graphically showed in Fig.2. More details on developed analysis procedure can be found in the Ref. [8].

Fig. 2 Typical MPPC pulse with its main characteristics.

The MPPC pulse characteristics allowed the calculation of important detector parameters required to be well known when such detectors are used in any application or experience. Between these parameters, some of them such as gain, breakdown voltage, signal shape, quenching resistance and capacitance, dark count rate are reported in the following. However, the analysis of acquired data requires more additional work (i.e. analysis of primary and secondary pulses of dark count rate, photon detection efficiency) and additional results will be reported in the future.

\footnotetext{
2 "single pulse": a MPPC signal separated by the neighboring pulses by a time interval higher than its recovery time

2 "train of pulses": two or more MPPC signals appearing with a time interval between them less than MPPC detector recovery time

${ }^{3}$ time between time position of the analyzed pulse and the previous one

${ }^{4}$ time between time position of the analyzed pulse and the next one
} 
The gain $\mathrm{G}$ of an MPPC is defined as a number of charges created in one avalanche in one $\mu$ cell and it can be experimentally determined from the time integration of device pulse presented in the Fig. 2:

$$
G=\frac{Q}{q_{e}}=\frac{\int I(t) d t}{q_{e}}=\frac{\int V_{\text {out }}(t) d t}{R_{\text {load }} \cdot G_{\text {amplifer }} \cdot q_{e}}
$$

where $\mathrm{Q}$ is the charge developed in one avalanche (i.e. 1 p.e. charge), $\mathrm{q}_{\mathrm{e}}$ is the electron charge, $\mathrm{I}$ is the current flowing from the detector to the readout circuit, $\int V_{\text {out }}(t) d t$ is the area of the signal, $\mathrm{G}_{\text {amplifier }}(50 \mathrm{~dB})$ is the gain of the amplifier, $R_{\text {load }}(50 \mathrm{Ohm})$ is the input amplifier load resistance.

The $\mathrm{G}$ as a function of $\mathrm{V}_{\text {bias }}$ for different $\mathrm{T}$ has been calculated for both measured detectors and they showed similar dependence of $\mathrm{V}_{\text {bias }}$ and $\mathrm{T}$. Therefore, for simplicity, only the results corresponding to $1 \times 1 \mathrm{~mm}^{2}$ detector are reported in Fig.3. We can observe that, at a given $\mathrm{T}, \mathrm{G}$ increases linearly with $\mathrm{V}_{\text {bias }}$ as expected from the gain equation:

$$
G=\frac{Q}{q_{e}}=\frac{C_{\mu c e l l} \cdot\left(V_{\text {bias }}-V_{B D}\right)}{q_{e}}
$$

with a slope proportional to the $\mu$ cell capacitance $\mathrm{C}_{\mu \text { cell }}$. However, if the temperature decreases while keeping $\mathrm{V}_{\text {bias }}$ constant, the $\mathrm{G}$ increases of $\sim 6 \% /{ }^{\circ} \mathrm{C}$, determining important variations of the input charge to the readout electronics if the $\mathrm{T}$ changes. This $\mathrm{G}$ variation is directly related to $\mathrm{T}$ variation of $\mathrm{V}_{\mathrm{BD}}$, as will be shown in the following section.

Fig. 3 Gain vs. $V_{\text {bias }}$ at different $\mathrm{T}$ for $1 \times 1 \mathrm{~mm}^{2}$ detector.

\subsection{Breakdown voltage}

Since no avalanches take place before breakdown, the $\mathrm{V}_{\mathrm{BD}}$ can be calculated as the intersection of linear fits presented in Fig.3 with abscise axis. The $\mathrm{V}_{\mathrm{BD}}$ as a function of $\mathrm{T}$ for both measured detectors is presented in Fig.4.

It can be observed that $\mathrm{V}_{\mathrm{BD}}$ of $1 \mathrm{x} 1 \mathrm{~mm}^{2}$ detector has a higher $\mathrm{T}$ dependence, decreasing of $12 \mathrm{~V}$ (i.e. $\mathrm{V}_{\mathrm{BD}} @+55^{\circ} \mathrm{C}=70 \mathrm{~V} ; \mathrm{V}_{\mathrm{BD}} @-175^{\circ} \mathrm{C}=58 \mathrm{~V}$ ) with respect to $3 \times 3 \mathrm{~mm}^{2}$ detector for which $\mathrm{V}_{\mathrm{BD}}$ decreases of only $9.5 \mathrm{~V}$ in the same $\mathrm{T}$ range (i.e. $\mathrm{V}_{\mathrm{BD}} @+55^{\circ} \mathrm{C}=71 \mathrm{~V} ; \mathrm{V}_{\mathrm{BD}} @-175^{\circ} \mathrm{C}=61.5 \mathrm{~V}$ ). We should remember here that the $1 \times 1 \mathrm{~mm}^{2}$ detector has been produced in 2007 , while the $3 \times 3 \mathrm{~mm}^{2}$ in 2011 , showing that recent MPPC devices have lower $\mathrm{T}$ dependence of $\mathrm{V}_{\mathrm{BD}}$ with respect to old ones.

An offset of $1 \mathrm{~V} @+55^{\circ} \mathrm{C}$ and $3.5 \mathrm{~V} @-175^{\circ} \mathrm{C}$ can be observed between the $\mathrm{V}_{\mathrm{BD}}$ values of measured detectors. In agreement with Ref. [9] this offset can be associated to a difference between the structural or technological parameters of measured detectors (e.g. background doping concentrations).

The $\mathrm{V}_{\mathrm{BD}}$ values of $1 \mathrm{x} 1 \mathrm{~mm}^{2}$ detector calculated from reverse IV characteristics by using the second derivative are also reported in Fig.4 (circles symbols). A difference of $0.5 \mathrm{~V}$ is found between the $\mathrm{V}_{\mathrm{BD}}$ values determined by two methods, showing that using IV shapes to determine $\mathrm{V}_{\mathrm{BD}}$ should be used with precaution, since they can determine a systematic error in the absolute values of $\mathrm{V}_{\mathrm{BD}}$. 
Knowing $\mathrm{V}_{\mathrm{BD}}$, the overvoltage parameter defined as $\Delta \mathrm{V}=\mathrm{V}_{\text {bias }}-\mathrm{V}_{\mathrm{BD}}$ can be determined. Figs. 5 a) and b) present the $\mathrm{G}$ dependence of $\Delta \mathrm{V}$ for $1 \mathrm{x} 1 \mathrm{~mm}^{2}$ and respectively $3 \times 3 \mathrm{~mm}^{2}$ detectors. The $\mathrm{G}$ increases linearly with $\Delta \mathrm{V}$, as already described by the Eq. 2. At a given $\Delta \mathrm{V}$, it can be observed that $\mathrm{G}$ is almost constant, presenting a variation of $\sim 5 \%$ for $1 \times 1 \mathrm{~mm}^{2}$ detector and $\sim 8 \%$ for $3 \times 3 \mathrm{~mm}^{2}$ detector. These small variations over the wide range of $\mathrm{T}$ used in our measurements (i.e. $230^{\circ} \mathrm{C}$ ) probably reflect the systematic error of the procedure used in the analysis and are related to the variation of the pulse shapes and the external noise with the temperature. This is attested also by the fact that $\mathrm{C}_{\mu \text { cell }}$ (i.e. slope of $\mathrm{G}$ vs. $\Delta \mathrm{V}$ ) shows no particular dependence of $\mathrm{T}$, as presented in the following.

The $\mathrm{C}_{\mu \text { cell }}$ was calculated from the slope of the linear fits presented in Figs. 5 a) and b) and its temperature dependence is represented in Fig. 6. Mean values of $\mathrm{C}_{\mu \text { cell }}=71.5$ $\pm 0.1 \mathrm{fF}$ and $53.6 \pm 0.1 \mathrm{fF}$ have been determined for $1 \times 1 \mathrm{~mm}^{2}$ and respectively $3 \times 3 \mathrm{~mm}^{2}$ detectors. That $\mathrm{C}_{\mu \text { cell }}$ is related to $\mu$ cell geometry, through the plan parallel capacitance equation:

$$
C=\frac{\varepsilon_{S i} \varepsilon_{0} A}{d}
$$

where $\varepsilon_{0}=8.854 \times 10^{-14} \mathrm{~F} / \mathrm{cm}$ is the permittivity of the free space, $\varepsilon_{S i}=11.9$ is the silicon dielectric constant, $\mathrm{A}$ is the active area and $\mathrm{d}$ is depletion thickness of $\mu$ cells. Geometrical measurements performed on the detectors surface showed that the overall $\mu$ cell area (i.e. pitch) is $50 \times 50 \mu \mathrm{m}^{2}$ for both detectors, as indicated by the Hamamatsu data sheet. However, the $3 \times 3 \mathrm{~mm}^{2}$ detector shows a $\mu$ cell active window of $\sim 38 \times 38 \mu \mathrm{m}^{2}$, smaller of $\sim 18 \%$ than the one of the $1 \times 1 \mathrm{~mm}^{2}$ detector which is $\sim 42 \times 42 \mu \mathrm{m}^{2}$. Since the difference between measured $\mathrm{C}_{\mu \text { cell }}$ is $\sim 25 \%$, the remaining difference of $\sim 7 \%$ can be related to some structural or technological differences (i.e. influencing the depletion thickness or other intrinsic MPPC characteristics).

$$
\text { Fig. } 5 \text { Gain vs. } \Delta V \text { for a) } 1 \times 1 \mathrm{~mm}^{2} \text { and b) } 3 \times 3 \mathrm{~mm}^{2} \text { detectors. }
$$

\subsection{Signal shape}

Fig. $6 \mathrm{C}_{\mu \text { cell }}$ vs $\mathrm{T}$ for $1 \mathrm{x} 1$ and $3 \times 3 \mathrm{~mm}^{2}$ detectors.

The collection of normalized MPPC single pulses at different $\mathrm{T}$ is presented in Figs. 7 a) and b) for $1 \times 1 \mathrm{~mm}^{2}$ and $3 \times 3 \mathrm{~mm}^{2}$ detectors respectively. The shape of our pulses is slightly modified by our read-out electronics (i.e. $20 \mathrm{MHz}$ oscilloscope bandwidth). However, we can distinguish that each pulse is characterized by a fast leading edge and a slow exponential decay. In the limits of our experimental errors, rising edge shows no $\mathrm{T}$ dependence, while the exponential decay shows a full length $t_{\text {recovery }}$ is increasing from $\sim 35 \mathrm{~ns}$ to $\sim 230 \mathrm{~ns}$ for $1 \mathrm{x} 1 \mathrm{~mm}^{2}$ detector and from $\sim 45 \mathrm{~ns}$ to $\sim 160 \mathrm{~ns}$ for $3 \times 3 \mathrm{~mm}^{2}$ detector while $\mathrm{T}$ decreases from $+55^{\circ} \mathrm{C}$ to $-175^{\circ} \mathrm{C}$.

Using the electrical model of MPPC detectors [10], one can expect that $t_{\text {recovery }}=5 \cdot \tau$ is related to $\mu$ cell recovery time (i.e. the time required to recharge the diode to $99 \%$ of bias voltage), characterized by a time constant $\tau \sim \mathrm{C}_{\mu \text { cell }} \times \mathrm{R}_{\mathrm{q}}=\left(\mathrm{C}_{\mathrm{D}}+\mathrm{C}_{\mathrm{Q}}\right) \times \mathrm{R}_{\mathrm{q}}$, where $\mathrm{C}_{\mathrm{D}}$ is the 
$\mu$ cell diode capacitance and $\mathrm{C}_{\mathrm{Q}}$ is a parasitic capacitance, in parallel with $\mathrm{R}_{\mathrm{q}}$ (usually, $\mathrm{C}_{\mathrm{Q}}$ $<C_{D}$ ). Since $C_{D}$ and $C_{Q}$ are independent of $T$ (i.e. Fig. 6), the $T$ dependence of $\tau$ is determined by the $\mathrm{T}$ dependence of $\mathrm{R}_{\mathrm{q}}$ as it will be shown in the following.

Fig. 7 Normalized MPPC signal shapes vs. T for a) $1 \times 1 \mathrm{~mm}^{2}$ and b) $3 \times 3 \mathrm{~mm}^{2}$ detectors.

The $R_{q}$, calculated as $\tau / C_{\mu \text { cell }}$, is presented in Fig. 8 as a function of $T$ for both measured detectors. It can be observed that independent of measured detector, $R_{q}$ exhibits large variation with $\mathrm{T}$, with decreasing values when $\mathrm{T}$ increases (i.e. negative $\mathrm{T}$ coefficient). This dependence is related to $\mathrm{T}$ variation of polysilicon resistance, as given by the expression [11]:

$$
R_{q}=a+b \sqrt{T} \exp \left(\frac{c}{T}\right)
$$

where $\mathrm{a}, \mathrm{b}$ and $\mathrm{c}$ are coefficients related to processing parameters of polycrystalline silicon resistors (i.e. grain size, doping concentration, trap density). The $\mathrm{a}, \mathrm{b}$ and c were set as free coefficients and Eq. 4 was used to fit $\mathrm{R}_{\mathrm{q}}$ as a function of $\mathrm{T}$ for both detectors. A good agreement can be observed between the experimental data and the fit as shown in Fig. 8.

Fig. $8 \mathrm{R}_{\mathrm{q}}$ vs. $\mathrm{T}$ for $1 \mathrm{x} 1 \mathrm{~mm}^{2}$ and $3 \times 3 \mathrm{~mm}^{2}$ detectors.

\subsection{Dark count rate}

The dark count rate DCR represents the number of avalanches per second generated by carriers traversing the depleted region when the device is kept in dark conditions. It is an important parameter because it sets a limit on the minimum detectable signal of a given device. The DCR includes primary (uncorrelated) and secondary (correlated) pulses. Primary dark pulses are mainly due to carriers thermally generated in the depletions regions of MPPC $\mu$ cells. Secondary dark pulses are related to afterpulsing and crosstalk effects.

From experimental point of view, the DCR can be determined by counting the MPPC pulses with amplitude exceeding a threshold of $0.5 \times 1$ pe amplitude. To avoid the light influence, the DCR was calculated using only $2 \mu \mathrm{s}$ time window of each experimental waveform, before the laser moment arrival.

The DCR as a function of $\Delta \mathrm{V}$ for different $\mathrm{T}$ are presented in Fig. 9 a) and b) for $1 \mathrm{x} 1$ $\mathrm{mm}^{2}$ and respectively $3 \times 3 \mathrm{~mm}^{2}$. It can be observed that at a given $\mathrm{T}$, the DCR increases exponentially with $\Delta \mathrm{V}$, because of $\Delta \mathrm{V}$ influence on different contributing probabilities (i.e. triggering probability, afterpulses and cross-talk probability). At a $\Delta \mathrm{V}=1.5 \mathrm{~V}$, the DCR drops of 1033 times for $1 \times 1 \mathrm{~mm}^{2}$ and 433 times for $3 \times 3 \mathrm{~mm}^{2}$ detector while $\mathrm{T}$ decreases from $+55^{\circ} \mathrm{C}$ to $-25^{\circ} \mathrm{C}$. Comparing the DCR $/ \mathrm{mm}^{2}$ (i.e. DCR depends on active area) between measured detectors at $\mathrm{T}=+25^{\circ} \mathrm{C}$ and $\Delta \mathrm{V}=1 \mathrm{~V}$, it can be observed that $1 \mathrm{x} 1$ $\mathrm{mm}^{2}$ detector shows $141,4 \mathrm{kHz} / \mathrm{mm}^{2}$, while the $3 \times 3 \mathrm{~mm}^{2}$ detectors shows $\sim 7$ times less $\left(19,8 \mathrm{kHz} / \mathrm{mm}^{2}\right)$. This result emphasizes again the improvements of MPPC technology between 2007 and 2011 . 


\section{Acknowledgments}

The authors would like to thank to Dr. Paul Rubinov and Dr. Donna Kubik for their help in the experimental set-up and data taking at Silicon Detector Facility (SiDet), Fermilab, USA. This work was granted by the Linear Accelerator Laboratory (LAL), University Paris XI and P2IO French Labex. Fermi National Accelerator Laboratory is operated by Fermi Research Alliance, LLC under Contract No. De-

\section{References}

[1] V.M.. Golovin, et al, (1999) , Russian patent, nr. 1644708

[2] B. Dolgoshein, P. Buzhan, A. Ilyin, V. Kantserov, V. Kaplin, A. Karakash, A Pleshko,. E. Popova, S. Smirnov, Y. Volkov, (2001), An Advanced study of silicon photomultiplier, ICFA Instrumentation Bulletin

[3] N. Dinu (October 2013); Instrumentation on Silicon Detector : from properties characterization to applications, LAL-13-192.

[4] K.K. Ng, S.M. Sze, Physics of semiconductor devices, Wiley, 2006

[5] M.J. Dean, et al., Low temperature electronics, Academic Press, 2001

[6] N. Serra, et al., (2011) TCAD simulation of avalanche breakdown voltage in GM-APDs. In: Proceedings of Nuclear Science Symposium Conference Record (NSS/MIC). Knoxville, TN: IEEE, 2010, 253-259

[7] www.root.cern.ch

[8] A. Nagai, (2013) Silicon Photomultiplier for Medical Imaging -Analysis of SiPM characteristics-, JRJC, 273 p43-46

[9] C.R. Crowell and S.M.Sze; (1966); Temperature dependence of avalanche multiplication in semiconductors,

275 Applied Physics Letters, Vol. 9, Issue 6, 242-244.

276 [10] S. Seifert, et. al , (2009) Simulation of silicon photomultiplier signals, IEEE Transactions on Nuclear 277 Science, Vol. 56, Issue 6, 3726-3733 
[11] N. C.C. Lu, et al. (1981) IEEE Trans. Electron Devices, 28, 818 
FigureV

Figure

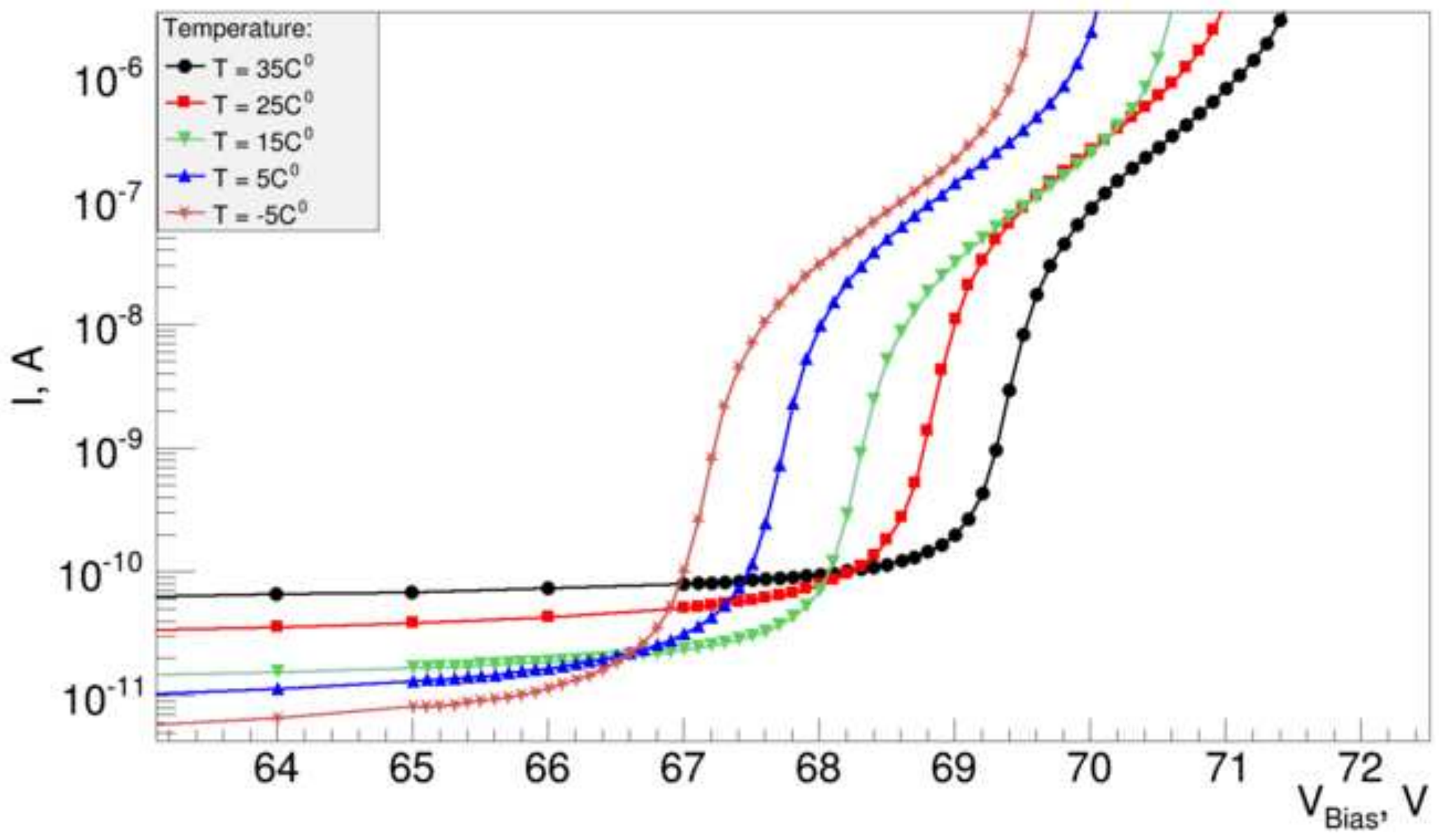




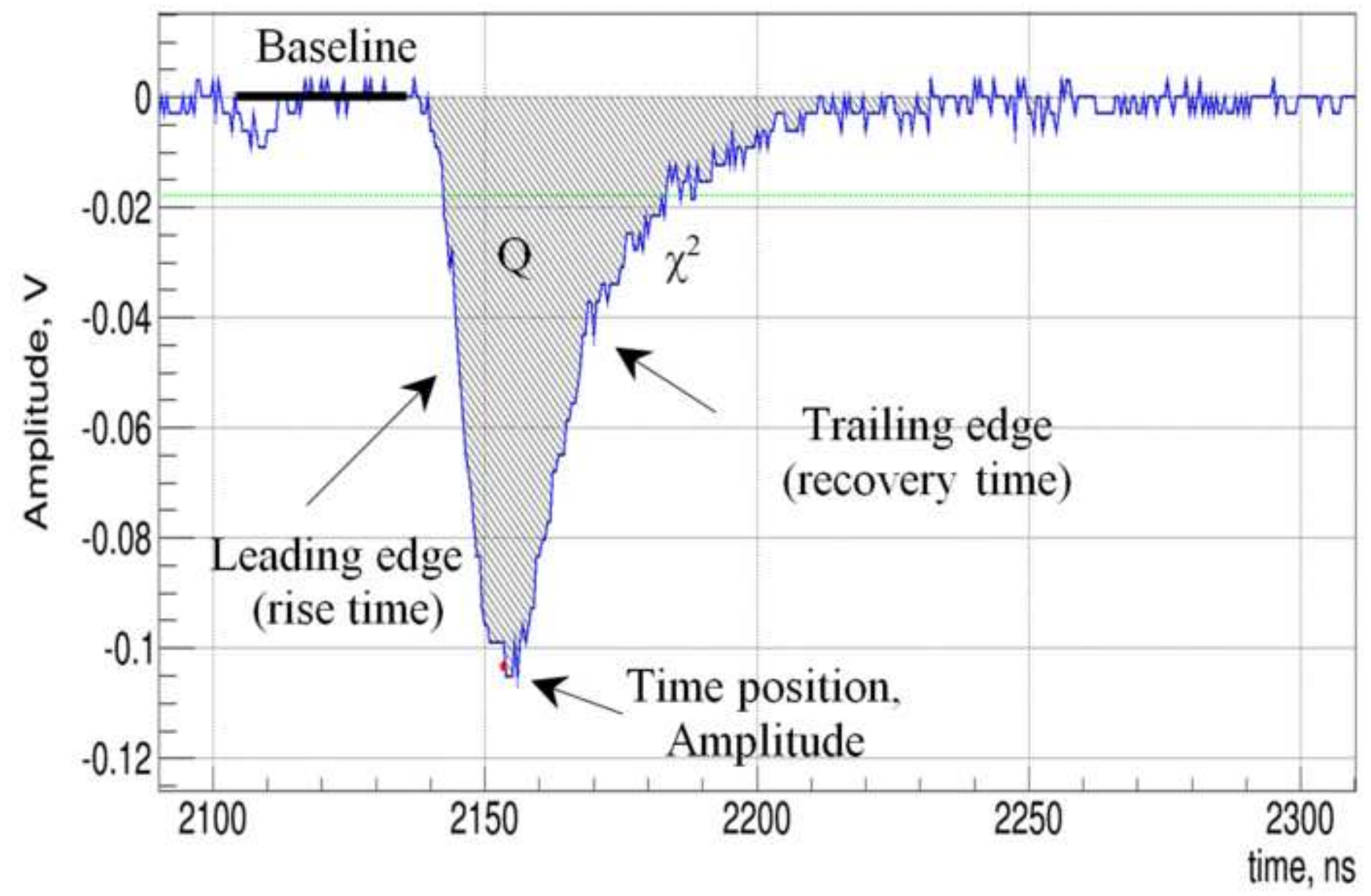




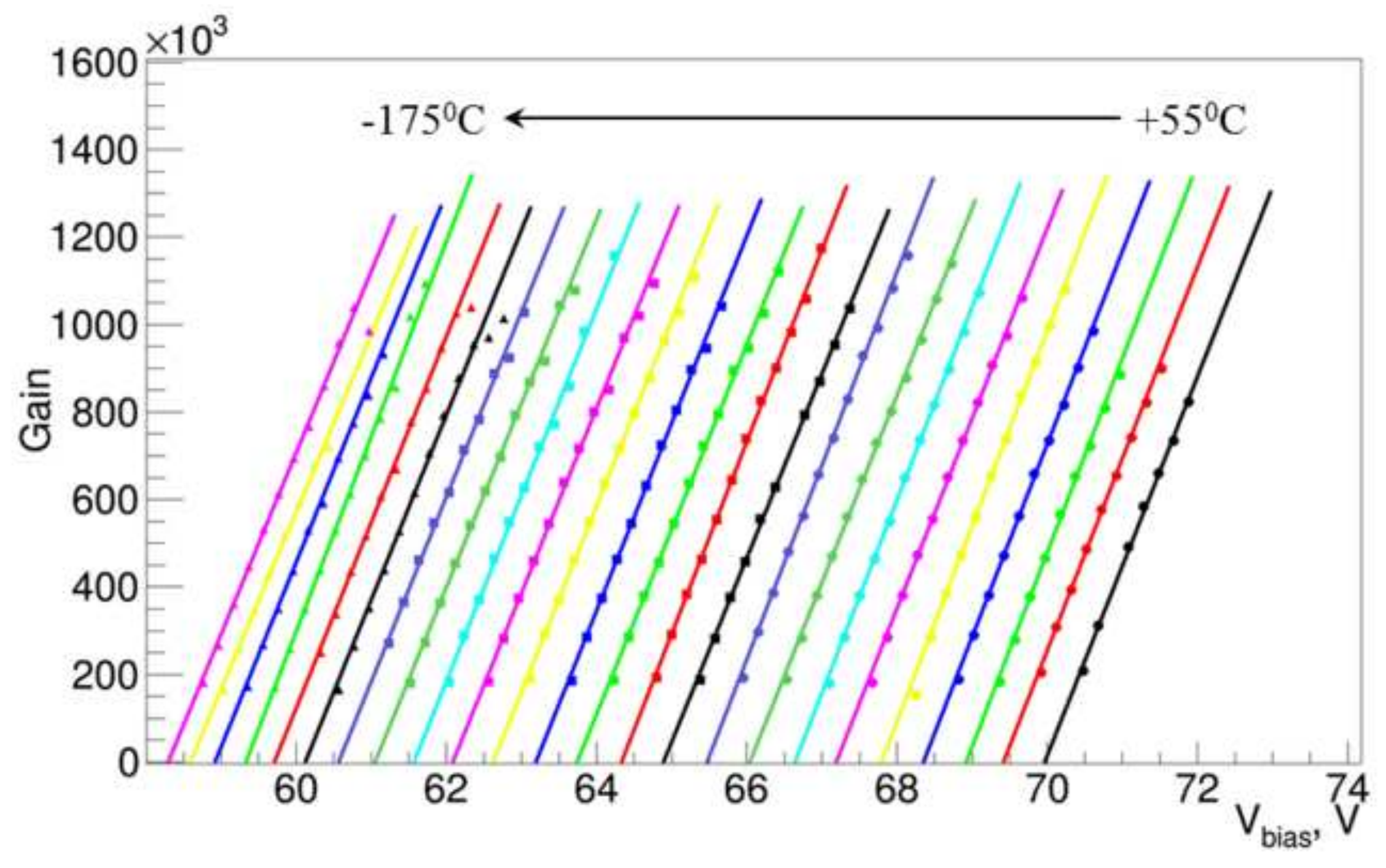




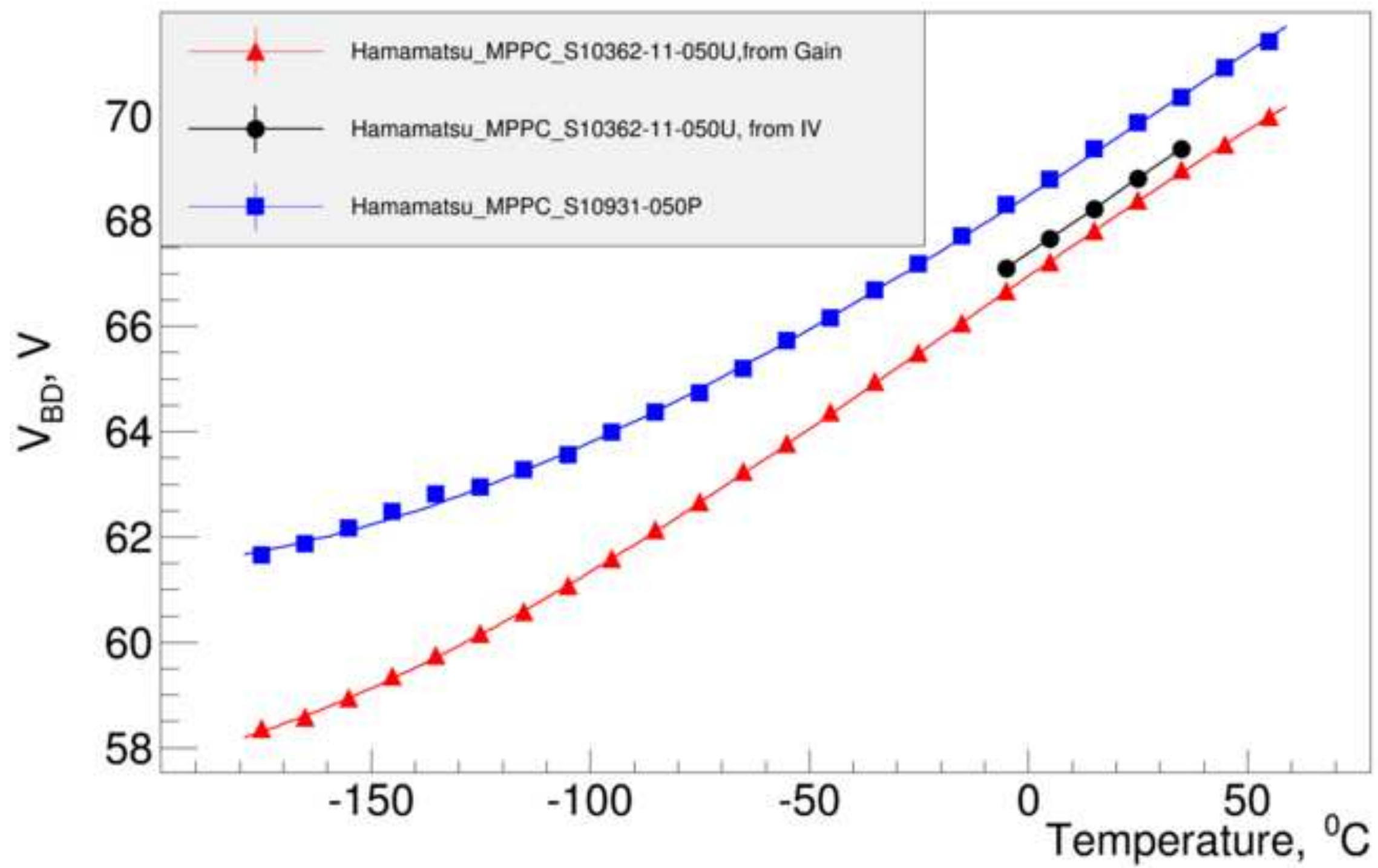




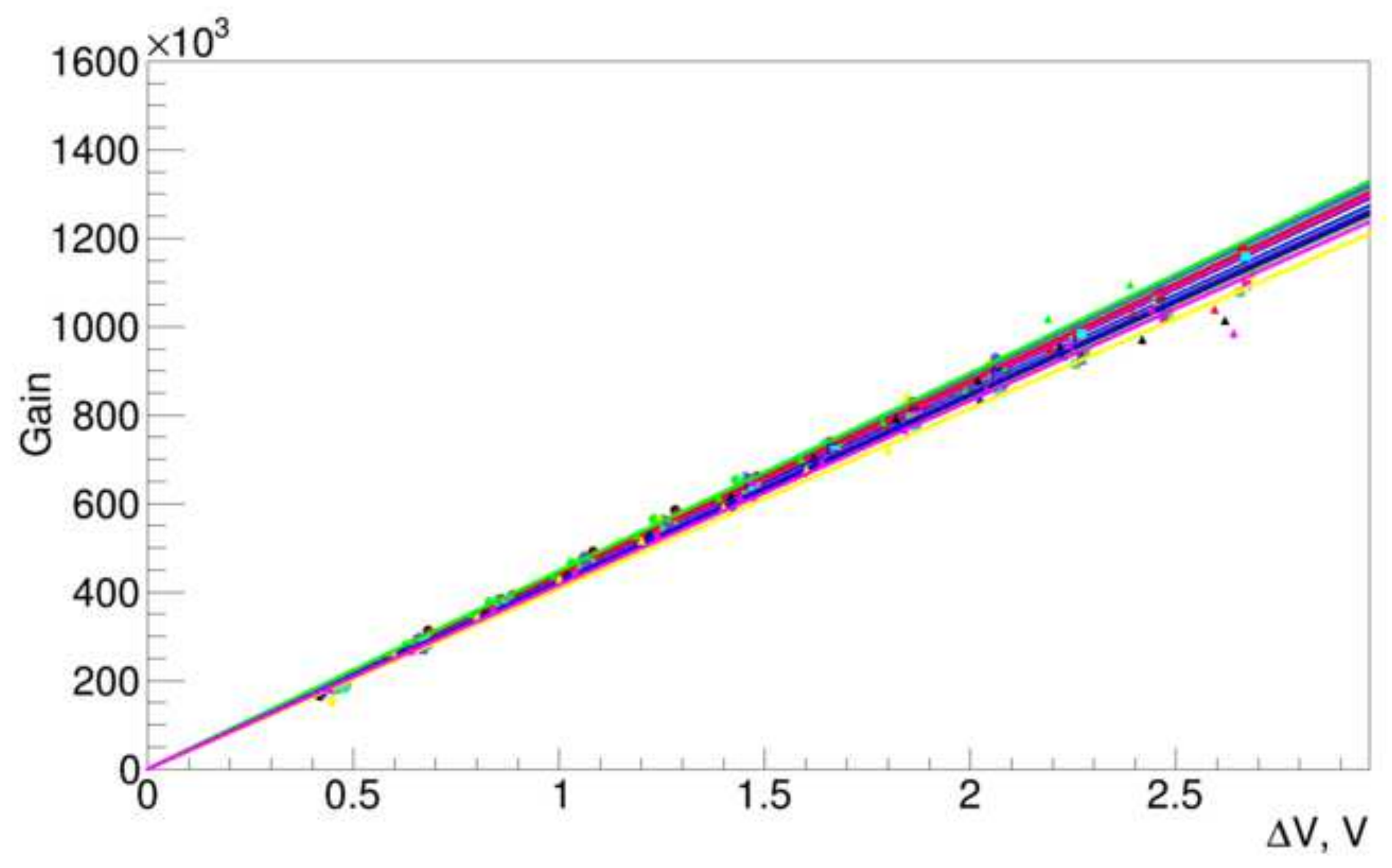




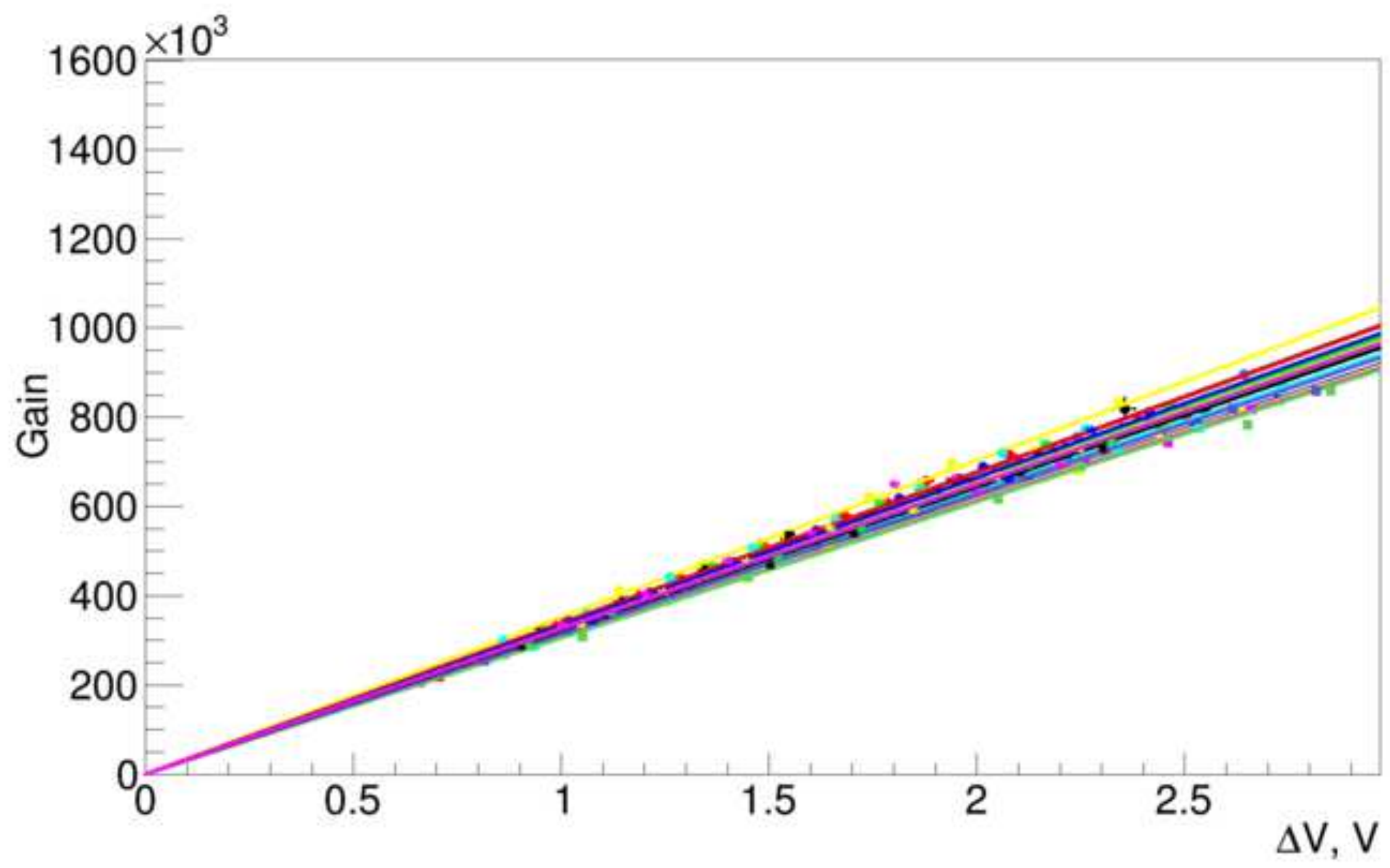




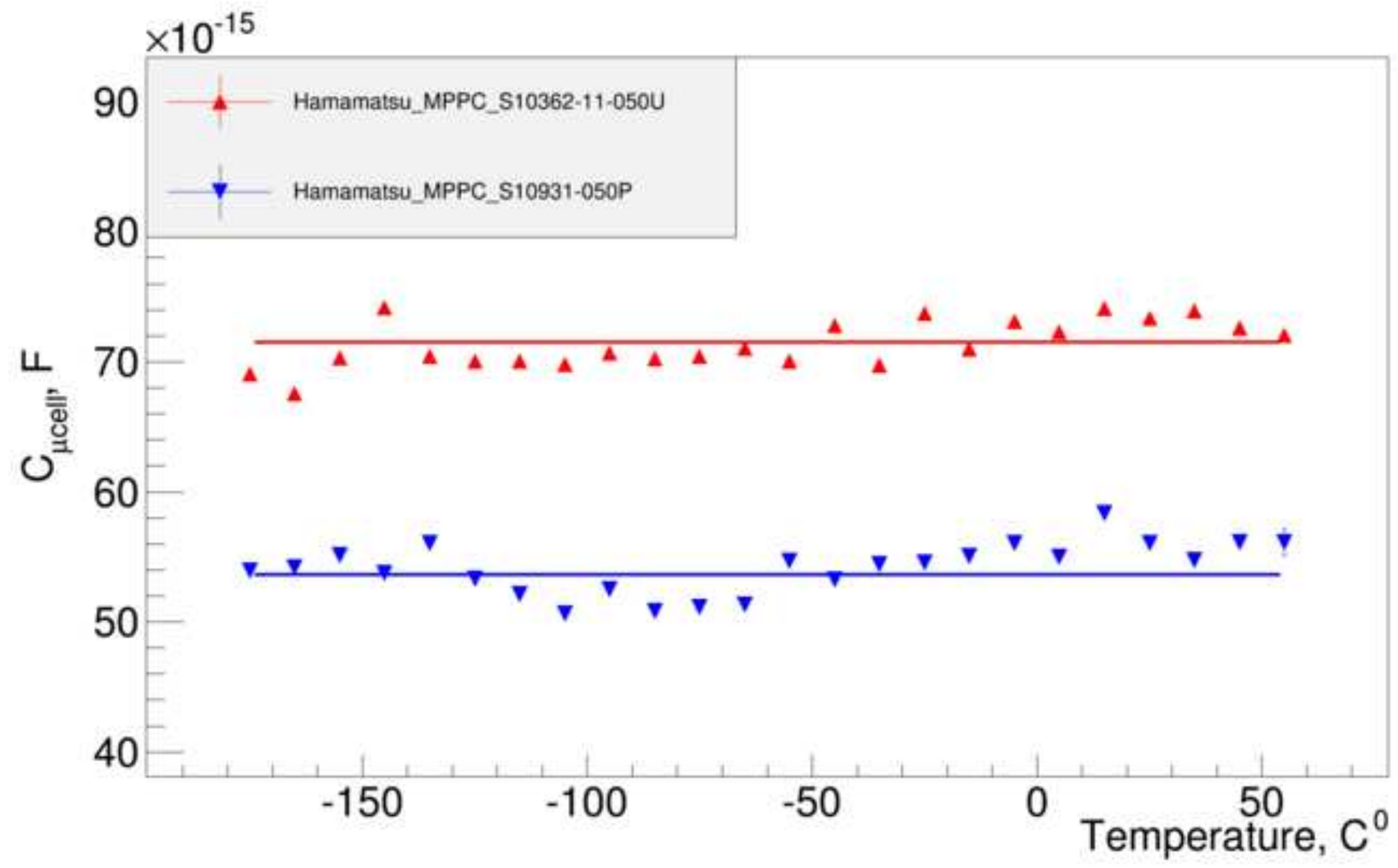




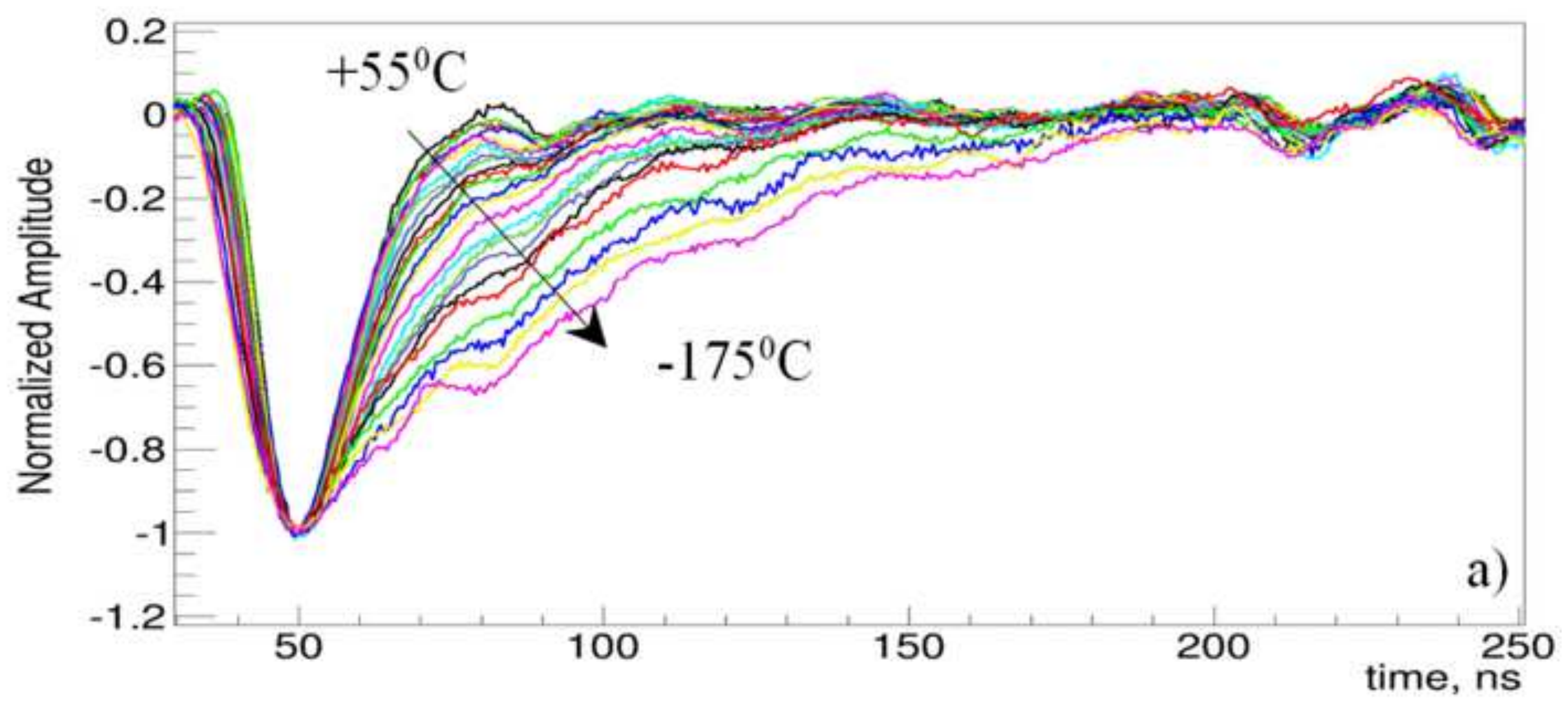




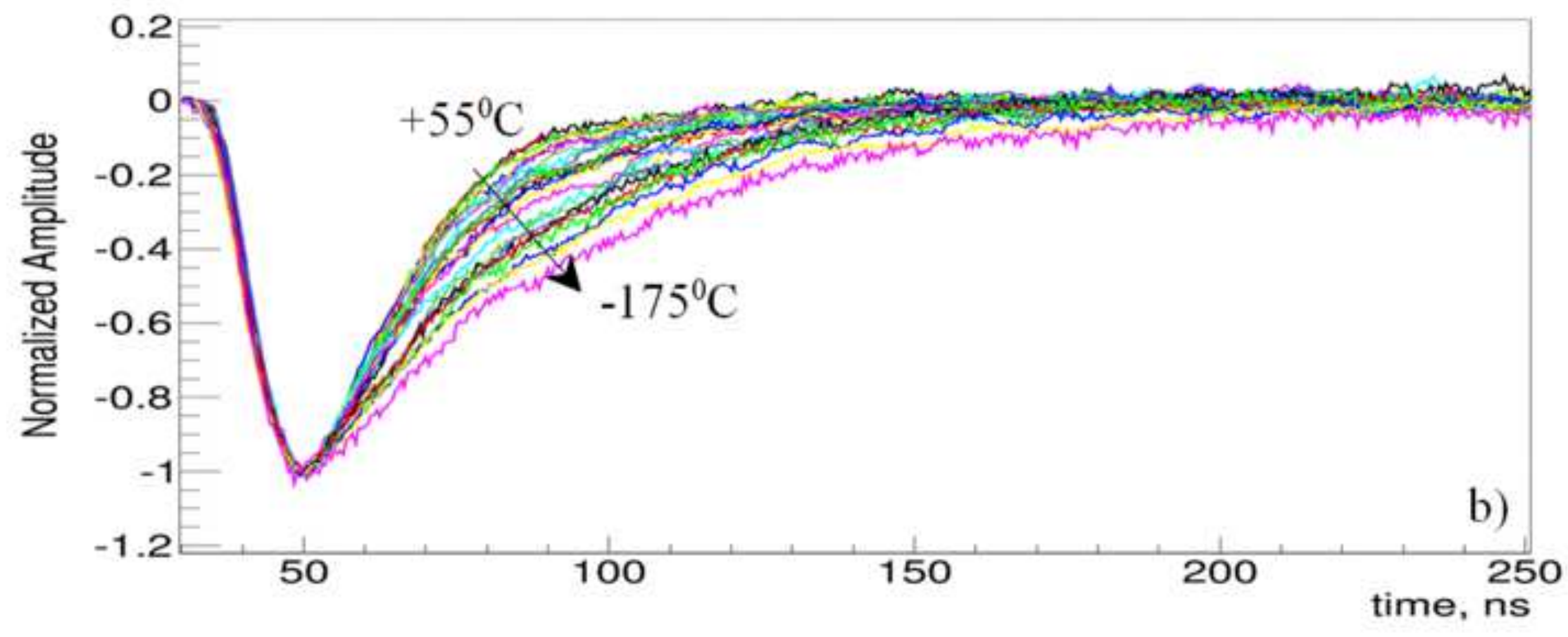




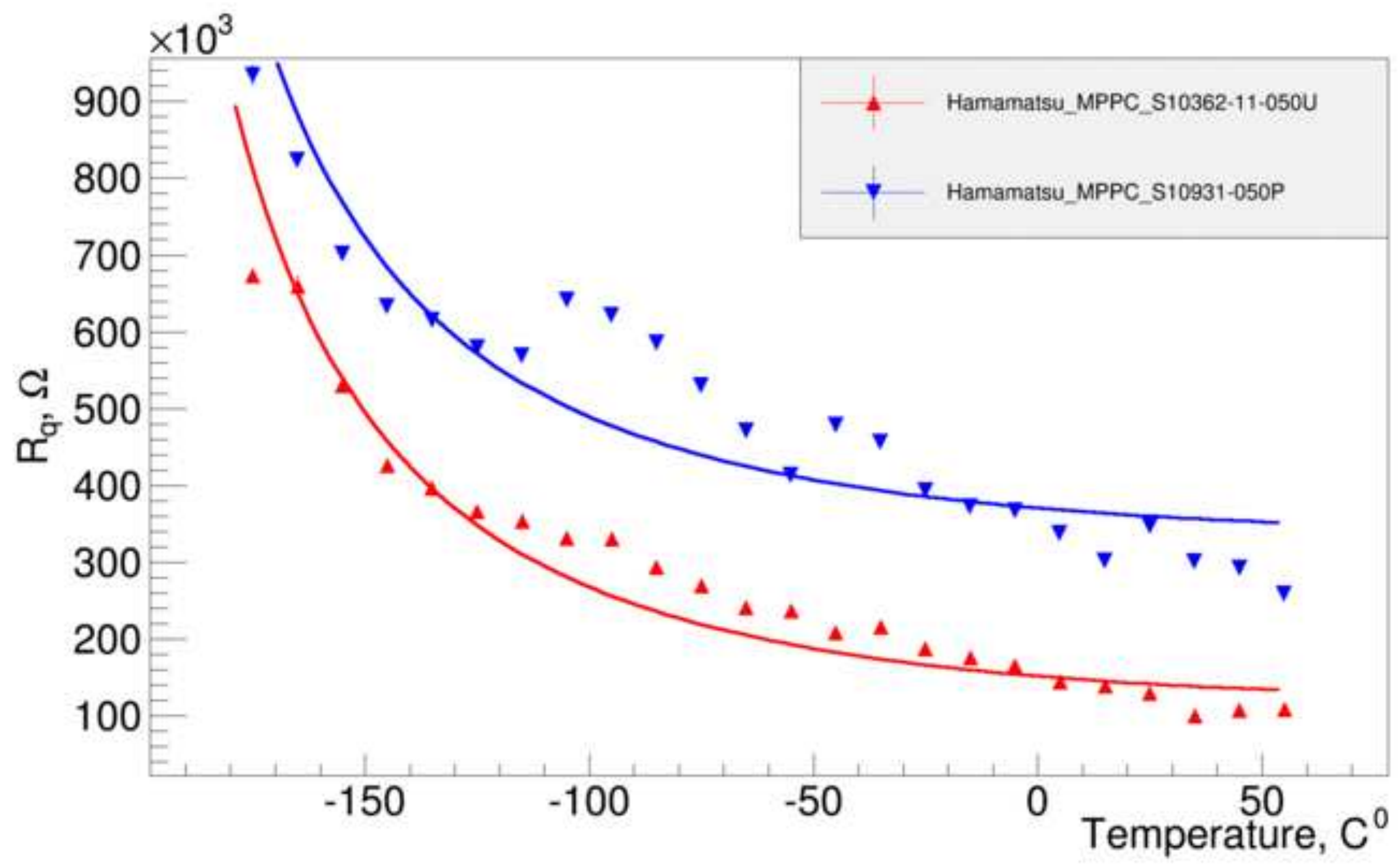




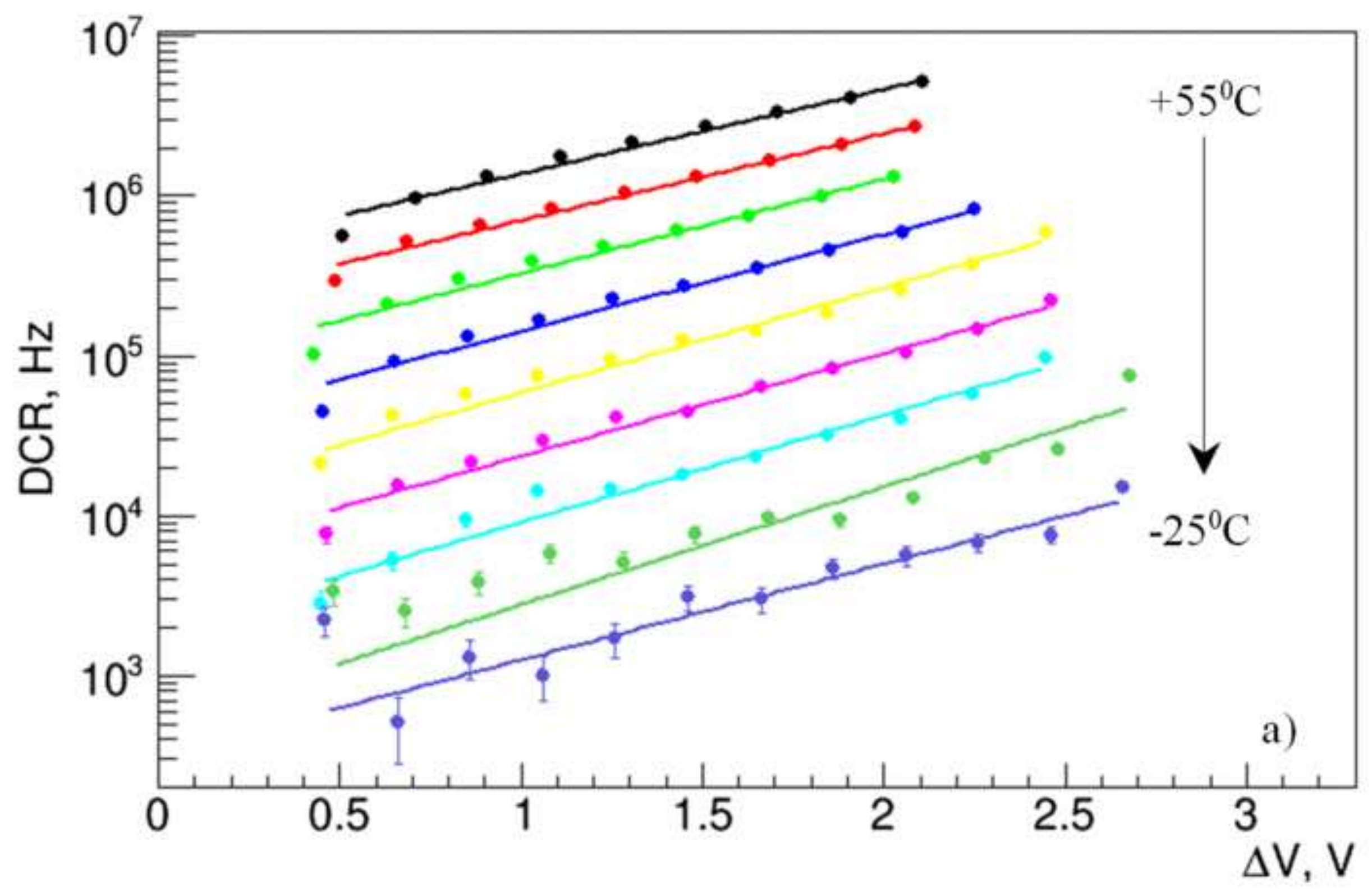




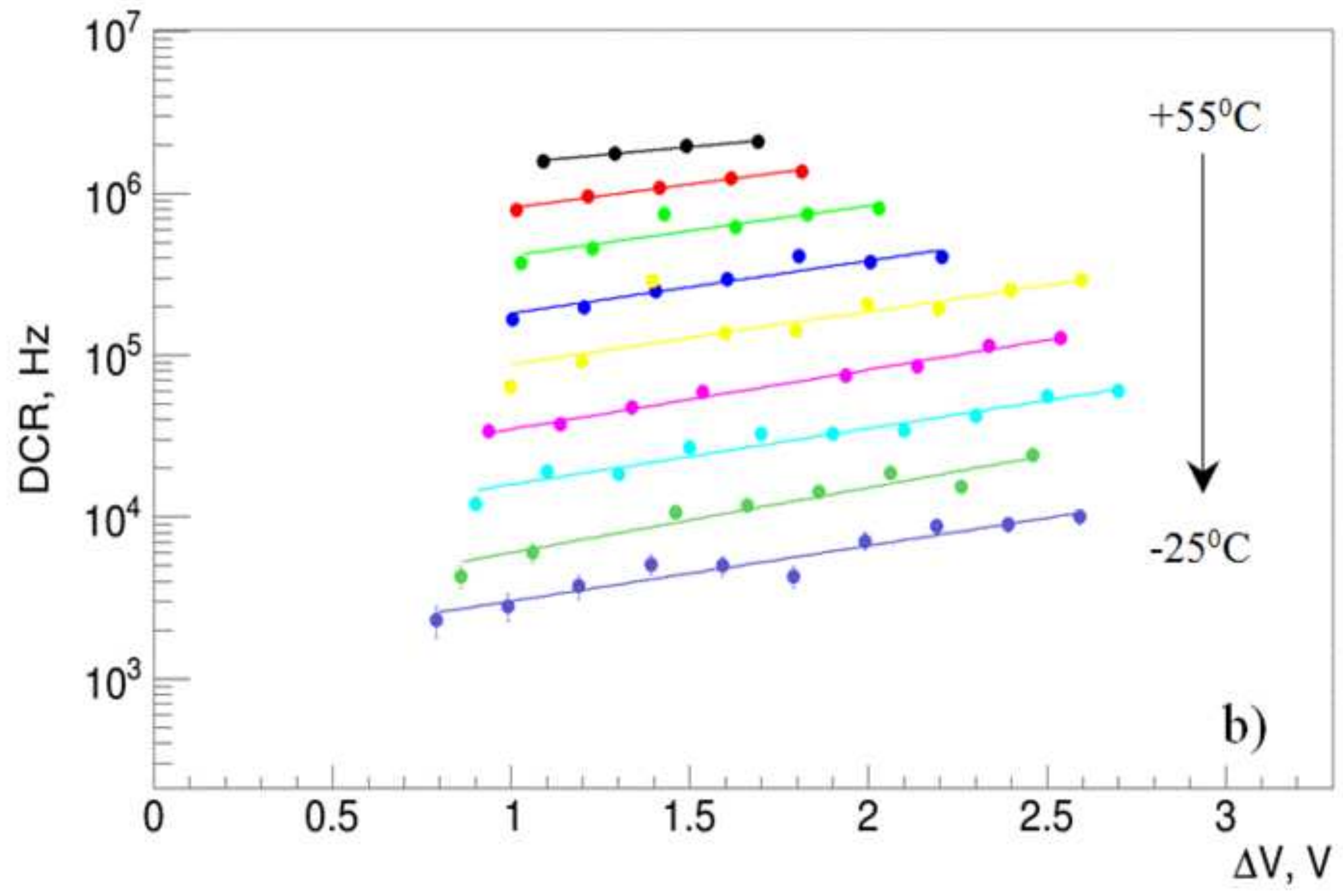

